\title{
Perikarditis
}

\section{Colchicin halbiert das Rezidivrisiko}

\author{
Bei rezidivierender Perikarditis \\ kann eine Behandlung mit Colchicin \\ zusätzlich zu NSAR die Heilung be- \\ schleunigen und das Rezidivrisiko \\ halbieren.
}

- Perikarditiden neigen zum Rezidivieren: bei $20-30 \%$ der Herzbeutelentzündungen kommt es zu Rückfällen, was zu erneuter Krankheit, Hospitalisation und Kosten führen kann. Zur Behandlung rezidivierender Perikarditiden empfiehlt die ESC Colchicin, obgleich bisher nur Daten aus nicht randomisierten und offenen Studien vorliegen.
Nun haben italienische Autoren erstmals eine doppelblinde Studie mit 120 Patienten durchgeführt, die ein erstes Perikarditis-Rezidiv erlitten hatten. Behandelt wurde sechs Monate lang mit NSAR plus Placebo oder Colchicin (Gewichtsabhängig 1-2 mg/d am 1 . Tag, danach $0,5-1 \mathrm{mg} / \mathrm{d})$. Primärer Endpunkt war die Rezidivrate nach 18 Monaten.

\section{Drei von vier Patienten}

nach drei Tagen wieder gesund

Der Mitose-Hemmstoff erwies sich als sehr wirksam: 55\% der Patienten in der Kontrollgruppe erlitten ein Rezidiv, aber nur 24\% in der Colchicin-Gruppe. Auch akut war das Gichtherapeutikum sehr wirksam: Nach nur drei Tagen waren die Symptome bei $77 \%$ der Patienten unter Colchicin abgeklungen, jedoch nur bei 47\% der Patienten der Kontrollgruppe. $5 \%$ vs. $13 \%$ der Patienten wurden erneut hospitalisiert. Zu leichten Nebenwirkungen kam es in beiden Gruppen in $7 \%$ der Fälle, wobei gastrointestinale Beschwerden dominierten.

$\mathrm{DE}$.

- ESC-Hotline 1, 28. 8. 2011; M. Imazio et al. Ann Intern Med 2011; published online 28.8.2011

\section{Hausärzte für Engagement zur Compliance-Verbesserung ausgezeichnet}

\begin{abstract}
Beim Europäischen Kardiologenkongress wurde der erste deutsche TherapieTreuePreis verliehen. Mit dem auf Initiative von Novartis ins Leben gerufenen Preis wurden vor 200 Gästen drei niedergelassene Kollegen geehrt, die sich in besonderem Maß für eine erfolgreiche Langzeittherapie ihrer Hypertoniepatienten einsetzen.
\end{abstract}

— "Drugs don't work in patients that don't take it": Gerade beim oft symptomlosen Bluthochdruck besteht die ärztliche Herausforderung, den Patienten von der langfristigen und konsequenten Behandlung zu überzeugen, betonte der Jury-Vorsitzende Prof. Rainer Düsing, Bonn.

\section{Viel Zeit, Empathie und Therapieüberwachung}

Für den TherapieTreuePreis 2011 hatten sich 71 Praxisteams aus ganz Deutschland beworben. Drei Kollegen schafften es ins Finale nach Paris. Der geteilte zweite Platz ging zum einen an die Internistin Irmgard Landgraf aus Berlin Steglitz, die in ihrer Praxis eine besonders gesprächs- und zeitintensive Hochdrucksprechstunde etabliert hat und die Tabletteneinnahme ihrer Patienten mit einer eigens entwickelten Medikamentensoftware überwacht. Ebenfalls mit dem 2. Platz ausgezeichnet wurde die Praxis von Dr. Franz Mader aus Miesbach. Er pflegt zu seinen Hochdruckpatienten ein besonders intensives Arzt-Patienten-Verhältnis unter Einbeziehung der Familienangehörigen und sortiert seinen Patienten die Medikation auch mal selbst in deren Tablettenspender, um die Einnahme zu erleichtern.

\section{Erster Preis an ein Praxisteam aus Hessen}

Der erste Preis ging an das Praxisteam des Facharztes für Allgemeinmedizin und Diabetologie Dr. Frank Klein und des Internisten Dr. Herrmann Finck aus Schenklengsfeld in Hessen. Gewürdigt wurden von der Jury v. a. die strukturierten und häufig durchge- führten Patientenschulungen unter Einsatz des Schulungsprogrammes HBSP, die eigens eingerichtete Hypertonie-Sprechstunde, die Bereitstellung einer Hypertonie-Assistentin DHL sowie die Entwicklung eines selbst entworfenen Messprotokolls in der Blutdrucklangzeitmessung.

\section{Compliance im Mittelpunkt}

„Die Hypertonie ist bei Novartis ein besonderer Forschungsschwerpunkt. Seit vielen Jahren stellen wir wirksame und sehr gut verträgliche Therapeutika zur Verfügung und tragen Sorge, dem Patienten die Therapie mit modernen Fixkombinationen so einfach wie möglich zu gestalten. Doch das alles nützt wenig ohne das tägliche Engagement der Ärzte, ihre Patienten von der Therapienotwendigkeit $\mathrm{zu}$ überzeugen. Deshalb haben wir die Compliance in den Mittelpunkt dieses neuen Preises gestellt, “ erklärte NovartisDeutschland-Chef Dr. Dirk Kosche, der in Paris den TherapieTreuePreis überreichte.

DE - 